\title{
Estudio exploratorio del bullying en Medellín
}

\author{
Viviana González Rodríguez ${ }^{\star}$, Jorge Iván Mariaca Patiño ${ }^{\star \star}$, Jacinta Lucía Arias Tobón ${ }^{\star \star \star}$
}

* Magíster en Avances e Investigaciones en Psicología de la Salud, Evaluación y Tratamiento Psicológico. Docente orientadora, Secretaría de Educación de Antioquia, Medellín, Colombia.

Correo electrónico:

webvital@gmail.com

** Comunicador Social, Universidad Pontificia Bolivariana, Medellín, Colombia. Tallerista en habilidades para la vida, jóvenes y adultos, Medellín, Colombia.

Correo electrónico:

jimariaca@misena.edu.co

*** Candidata a Magíster en Psicología Social, Universidad Pontificia Bolivariana, Medellín, Colombia. Psicóloga del Proyecto de Acompañamiento Psicosocial a la División de Menores del Deportivo Independiente Medellín (Equipo del Pueblo S. A.), Universidad Pontificia Bolivariana, Medellín, Colombia.

Correo electrónico:

jacinta.arias@upb.edu.co

Recibido: 8 de abril del 2014

Aprobado: 3 de septiembre del 2014

Cómo citar este artículo: González, V., Mariaca, J. I. y Arias J. L. (2014). Estudio exploratorio del bullying en Medellín. Pensando Psicología, 10(17), 17-25. doi: http://dx.doi.org/10.16925/pe.v10i17.776

\section{Resumen}

Este es un estudio exploratorio sobre el acoso escolar (o bullying) entre estudiantes de Medellín. Para adelantarlo se realizaron 3.373 encuestas virtuales en 36 centros de educación básica secundaria y media, con el fin de identificar la presencia del fenómeno del acoso escolar y de establecer las formas específicas de su manifestación teniendo en cuenta edad, género y grado escolar. Los resultados demostraron la presencia de bullying en el 37,6\% de los estudiantes encuestados, expresado en comportamientos de intimidación o agresión verbal, física y psicológica en estudiantes de ambos géneros, de todos los grados escolares. La forma de agresión de mayor frecuencia es la verbal y la psicológica, y estas suceden usualmente en presencia de pares y docentes en el aula de clase. Las principales formas de denominación de los estudiantes que ejercen bullying son agresor y abusador.

Palabras clave: acoso escolar, agresor, centros de educación básica secundaria y media, estudiantes, testigo, víctima.

\section{Exploratory Research Study of School Bullying in Medellin}

\section{Abstract}

This is an exploratory research study into school bullying among students in Medellin. To carry out the study, 3,373 virtual surveys were conducted across 36 secondary education centers, and this data was used to identify the presence of bullying and the specific forms it takes, according to variables such as age, gender and school grade. The results show that $37.6 \%$ of students across both sexes and across all school grades have come into contact with bullying, manifested in behavior such as intimidation and verbal, physical and psychological aggression. The most common forms of aggression were verbal and psychological, and these usually occurred in the presence of peers and teachers in the classroom. The main names given to students who engage in bullying are aggressor and abuser.

Keywords: school bullying, aggressor, secondary education centers, students, witness, victim.

\section{Estudo exploratório do bullying em Medellín}

\section{Resumo}

Este é um estudo exploratório sobre o bullying entre estudantes de Medellín (Colômbia). Para realizá-lo, foram aplicadas 3.373 enquetes virtuais em 36 centros de ensino fundamental e médio, a fim de identificar a presença do fenômeno do bullying e de estabelecer as formas específicas de sua manifestação considerando idade, gênero e grau escolar. Os resultados demonstraram a presença de bullying em 37,6\% dos estudantes pesquisados, expresso em comportamentos de intimidação ou agressão verbal, física e psicológica em estudantes de ambos os gêneros, de todos os graus escolares. A forma de agressão de maior frequência é a verbal e a psicológica, e estas acontecem usualmente em presença de pares e docentes na sala de aula. As principais formas de denominação dos estudantes que exercem bullying são agressor e abusador.

Palavras-chave: bullying, agressor, centros de ensino fundamental e médio, estudantes, testemunha, vítima 


\section{Introducción ${ }^{1}$}

El bullying es un fenómeno social que se presenta dentro de un contexto educativo, y que consiste en que uno o varios niños, niñas o adolescentes agreden física, verbal o psicológicamente a uno o varios de sus compañeros o compañeras.

Las consecuencias de este fenómeno para las víctimas incluyen el bajo rendimiento académico, la desmotivación escolar, los traumas psicológicos y los trastornos psicosomáticos; el bullying también es una de las causas de mayor deserción escolar. Esta acción puede generar en las víctimas descenso en su autoestima y mayores índices de ansiedad; asimismo, se les dificulta la integración en el medio escolar y el desarrollo normal de los aprendizajes (Osorio, 2009). Las secuelas para los agresores suelen ser las dificultades en la convivencia y las relaciones interpersonales; puede suponer para ellos un aprendizaje sobre cómo conseguir los objetivos y, por tanto, estar en la antesala de la conducta delictiva. Los agresores pueden conseguir un reforzamiento sobre el acto de dominio-sumisión como algo bueno y deseable; por otra parte, lo puede instrumentalizar como método para tener un estatus en el grupo, una forma de reconocimiento social por parte de los demás (Avilés, 2003a). Los testigos, por su parte, no permanecen libres de influencia respecto de estos hechos y pueden obtener un aprendizaje sobre cómo comportarse ante situaciones injustas y un refuerzo para posturas individualistas, egoístas y una valoración positiva de la conducta agresiva (Avilés, 2003b).

Debido a esto, en el ámbito internacional son varios los estudios realizados sobre el fenómeno del bullying. En los escritos de Thomas Hughes y Dan Olweus, se pueden encontrar investigaciones iniciales; desde entonces se han realizado estudios en escuelas de diversos países que confirman la existencia del problema y las consecuencias negativas que a corto y largo tiempo sufren quienes han padecido el problema en sus años escolares (Carrera, De Palma y Lameiras, 2011).

En la década de los ochenta e inicio de los noventa, investigadores de Finlandia, Estados Unidos, Inglaterra, Canadá, Japón, España, Países Bajos, Irlanda, Noruega, Suecia y Australia, a quienes cita Olweus (1998),

El artículo es producto de la investigación "Estudio exploratorio sobre del bullying en la ciudad de Medellín, Colombia", que se inició en mayo de 2012 y terminó en febrero de 2013. La investigación se hizo con los aportes de recursos financieros de la Corporación Educación Sin Fronteras, a quien los autores dan sus agradecimientos. obtuvieron datos que confirmaban la existencia del bullying en sus respectivos países, concluyendo que se presentaba en igual proporción a la de los estudios pioneros (5 a 10\%) o en muchos casos, superior; y en algunos países, hasta el $30 \%$ de estudiantes eran víctimas de agresiones frecuentes. También se encuentran datos de investigaciones transculturales que indican que el problema existe en proporciones similares -aunque con diferencias en su manifestación- aún en contextos étnicos diversos, con diferentes tradiciones y marcos culturales, como en Norteamérica, América Latina, Europa Oriental, Europa Occidental, Asia y Australia, principalmente (Paredes et al. 2011).

En el ámbito nacional, el acoso escolar es un fenómeno psicosocial que se presenta en menor o mayor medida en la mayoría de los centros de educación, como se muestra en las Pruebas Saber del Icfes del 2007, en las que se indagó sobre la presencia del matoneo escolar y se evidenció que, en el territorio nacional, un $22 \%$ de los estudiantes son víctimas, $21 \%$ son victimarios y $53 \%$ son testigos de matoneo (Rodríguez y Mejía, 2012). Otros estudios señalan la prevalencia del bullying en algunos centros de educación de ciudades como Montería, con un 69,2\% (Ávila, Osorio, Cuello, Cogollo y Causado, 2010); en el departamento de Santander, se encontró el 22,8\% de presencia de bullying en 304 estudiantes de grados sexto, noveno y undécimo (Uribe, Orcasita y Aguillón, 2012). En Bogotá, se halló que el 15\% de los estudiantes entre 7 y 10 años han sido víctimas de acoso escolar y que el $10 \%$ son niños agresores que generalmente han sufrido maltrato en su casa (Chaux, 2011). Mientras que, en los centros educativos públicos, el bullying es principalmente físico y verbal, en los privados crece el acoso virtual, y además los estudiantes de mayor edad en el curso, y los de estratos económicos más altos, generalmente son los agresores (Camargo, 2012).

En Cali, se realizó un estudio exploratorio del fenómeno del bullying (Paredes, Álvarez, Lega y Vernon, 2008), en el que se encontró una incidencia de este fenómeno en el 24,7\% de los encuestados. Además, se constató que se manifiestan todas las formas de agresión: física, psicológica y verbal, siendo esta última la más frecuente. En Barranquilla, por su parte, se aplicó una encuesta a estudiantes, en la cual se observa con más frecuencia que el bullying está iniciando en la adolescencia, sin embargo, la conducta disminuye a medida que aumenta la edad. Además, se encontró que la mayor forma de maltrato es verbal y quienes más agreden y son agredidos pertenecen al sexo femenino, siendo el aula de clase el lugar donde con más frecuencia suceden los actos de violencia escolar (Hoyos, 
Aparicio y Córdoba, 2005). En el departamento de Antioquia, específicamente, este fenómeno no es ajeno ya que tanto en la capital antioqueña, como en sus municipios, se han detectado casos en los grados académicos, con consecuencias negativas.

Esto ha preocupado al Ministerio de Educación Nacional, entidad que ha comenzado a realizar esfuerzos para disminuir el bullying entre sus estudiantes, haciendo énfasis en la importancia de generar buenos manuales de convivencia, fortalecer la promoción de la convivencia y prevenir la violencia en el ámbito escolar, creando para ello el Sistema Nacional de Convivencia Escolar y Formación para el Ejercicio de los Derechos Humanos, la Educación para la Sexualidad y la Prevención y Mitigación de la Violencia Escolar (Ley 1620 de 2013 y Decreto 1965 de 2013). Por esta razón, el siguiente trabajo tiene como objetivo realizar un muestreo exploratorio del fenómeno del bullying en Medellín, aportando indicadores de acoso escolar que tienen en cuenta los roles de las víctimas, los agresores y los testigos. Este trabajo aporta datos estadísticos que permiten reflexionar sobre la incidencia del bullying.

\section{Metodología}

\section{Descripción del estudio}

Este es un estudio exploratorio descriptivo que recoge datos de estudiantes de Medellín, una investigación cuantitativa de corte transversal. El primer paso para este estudio consistió en buscar un test que midiera el acoso escolar; para ello, en el 2012, se pidió autorización a las investigadoras Paredes, Álvarez, Lega y Vernon (2008) para utilizar el test que ellas diseñaron y aplicaron en Cali. No obstante, se hicieron dos modificaciones: la primera, es que el test original investiga a víctimas y victimarios, mientras que en este estudio se adicionan datos de los y las testigos; la segunda, es que se realizó de manera virtual y no presencial.

Posteriormente, se dictó una conferencia con los docentes y directivos docentes de los centros de educación básica secundaria y media, interesados en participar de la investigación, y se les brindó una introducción sobre lo que es el bullying, sus causas, consecuencias, estrategias de prevención e intervención. De igual manera, se expuso tanto la fundamentación teórica y metodológica de la investigación, como la manera de aplicar el test a sus estudiantes. Durante un mes, la encuesta estuvo publicada de manera virtual, y a ella accedían los estudiantes con la orientación de sus docentes. Tras obtener los datos, se realizó un filtro de las 36 instituciones participantes. Se hizo una devolución por escrito de los resultados a los centros de educación básica secundaria y media que participaron con más de 40 estudiantes en la encuesta.

\section{Muestreo}

Se realizó una muestra incidental no probabilística entre 36 centros de educación básica secundaria y media que expresaron su interés en participar del estudio. La técnica empleada en la prueba de campo fue la encuesta individual virtual aplicada a estudiantes de los grados sexto, séptimo, octavo, noveno, décimo y undécimo. Los grados se seleccionaron porque los estudiantes en estos grados tienen un manejo básico de computadores e Internet. La encuesta se realizó durante los meses de julio y agosto del 2012.

\section{Participantes}

Quienes respondieron como mínimo la mitad de la encuesta fueron 3.373 estudiantes, de los cuales 1.838 son mujeres $(54,5 \%)$ y 1.535 hombres (45,5\%). La edad de los estudiantes estuvo distribuida así: 7,98\% entre 9 y 11 años; 45,54\% entre 12 y 14 años; 40,29\% entre 15 y 17 años; 4,51\% entre 18 y 20 años, y 1,69\% más de 20 años. La distribución por grados fue la siguiente: grado sexto $(24,13 \%)$, grado séptimo $(17,85 \%)$, grado octavo $(16,04 \%)$, grado noveno $(16,81 \%)$, grado décimo $(13,58 \%)$ y grado undécimo $(11,59 \%)$; se observa disminución del número de participantes al aumentar el grado.

\section{Instrumento de recolección de datos}

Para identificar al agresor y a la víctima, se utilizó la encuesta diseñada y elaborada por Paredes et al. (2008). Las preguntas del instrumento se construyeron teniendo en cuenta los aspectos que definen el problema según Olweus (2001), que son: 1) que un alumno es víctima de bullying cuando es agredido de forma repetida e intencionada por uno o varios compañeros; 2) que las principales formas de agresión son golpes, amenazas, exclusiones y ridiculizaciones; 3) que debe existir una relación de poder asimétrica entre víctima y agresor; 4) que estas acciones negativas pueden ser evidentes o no evidentes; y 5) que tienen la finalidad general de identificar a los alumnos y alumnas que res- 
pondan en la situación de agresores, agresoras y a quienes responden en la situación de víctimas.

El test no refiere datos de fiabilidad y validez; se aplicó una prueba piloto con el objetivo de examinar el cuestionario y perfeccionar los aspectos logísticos de su aplicación (Paredes et al., 2008), y las preguntas realizadas son de tipo pregunta abierta, selección múltiple con única respuesta y selección múltiple con múltiple respuesta. Asimismo, cuenta con un encabezado que recoge la información general y sociodemográfica de los y las estudiantes. Debido a que en idioma español no existe un término que se refiera con exactitud al problema, se incluyó una pregunta para saber si los estudiantes y las estudiantes coinciden con un nombre particular para denominar a los compañeros y compañeras que llevan a cabo este tipo de acciones. Posteriormente, se preguntan aspectos subjetivos relacionados tanto con aquellas personas que cometen acciones agresivas con sus pares en el contexto escolar, como con la percepción de los que se consideran víctimas de este tipo de conductas.

\section{Técnicas de procesamiento y análisis de datos}

Se emplea la estadística descriptiva, en tanto se analiza y se representan los datos a través de Microsoft Excel. Se realiza la tabulación de los resultados en tablas básicas de cada una de las variables seleccionadas, acorde con las respuestas de los 38 ítems del cuestionario. Se analiza estadísticamente el total de respuestas brindadas a cada subgrupo de preguntas, ya sea en el rol de agresor, víctima o testigo; se establece correlación respecto a la frecuencia de presentación en cada una de ellas para señalar el porcentaje de acoso escolar en la población participante. Para esta investigación, se establecieron como indicadores de la presencia de bullying todas aquellas manifestaciones de agresión que se presentan con una frecuencia de diariamente, varias veces a la semana, varias veces al mes y, por lo menos, una vez al mes, teniendo en cuenta la conceptualización del fenómeno de bullying en relación con la violencia escolar, en tanto que el primero requiere una frecuencia constante en el tiempo, mientras que el segundo hace referencia a episodios ocasionales o incidentales de agresión (Paredes et al., 2008).

\section{Resultados}

Con el fin de presentar los resultados de acuerdo con la intención descriptiva del instrumento, se iniciará con la forma como los estudiantes denominan la agresión en el contexto educativo; posteriormente, se resaltarán los resultados de los estudiantes que reconocieron ser agresores, seguido de aquellos que han sido víctimas en sus instituciones, y se finaliza con aquellos que han sido testigos de acoso escolar.

\section{Denominación de la agresión}

En la investigación, se halló que en el ítem que pregunta a los estudiantes sobre cómo denominan a las personas en el colegio que golpean, amenazan, excluyen o ridiculizan a sus compañeros, se encontró que el $43,44 \%$ los denominan de la siguiente manera: agresor (15,4\%), abusador/abusivo (11,4\%), nombre propio $(5,4 \%)$, aprovechado $(4,4 \%)$, descarado $(3,7 \%)$ y bullying (3,1\%); mientras que el 56,56\% denomina a quienes agreden de manera diferente con calificativos y adjetivos negativos como "bravucón", "baboso", "animal", entre otros, o con denominaciones que le otorgan poder a los agresores como "caciques", "jefes" o "patrones". Sin embargo, por la alta dispersión de las respuestas, no se identifica una palabra exacta que nombre en la población hispanoparlante a quien ejerce el bullying.

\section{Aspectos de los agresores}

Para definir las características de quienes han agredido a un par en el contexto escolar, se tuvieron en cuenta las respuestas del cuestionario que evalúan la conducta de aquellos se reconocieron como agresores y agresoras. Se encontró que el 58,35\% de todos los encuestados y encuestadas admitió que alguna vez ha agredido de diferentes formas a un compañero o compañera, ridiculizándolo (28,4\%), golpeándolo (26,12\%), excluyéndolo $(9,4 \%)$ o por medio de la amenaza $(6 \%)$, y con dos o más de las anteriores formas de agresión (30,08\%). La agresión referida por los estudiantes ocurre en presencia de otros en un $64,28 \%$. En cuanto a las consecuencias evidentes de la conducta agresiva, se encuentra un porcentaje de $12,25 \%$ de estudiantes que señalan haber recibido alguna consecuencia a nivel institucional (anotación, ser llevado a las directivas, citación a padres de familia, seguimiento con debido proceso, llamado de atención, entre otras), y un 58,18\% responde que tuvo consecuencias variadas, especialmente referidas a enemistades, lesiones personales, amenazas verbales, con armas y sentimientos de culpabilidad. En relación 
con los sentimientos que emergen tras la agresión, los que tienen mayor índice son: la culpabilidad, con un $17,53 \%$, la preocupación con el 16,01\% y la satisfacción con el 13,06\%; asimismo, un 25,91\% expresó haber presentado dos o más emociones tras agredir.

Con base en el criterio de categorización antes descrito, del total de estudiantes que refirieron haber agredido a un compañero o compañera de clase, el 48,17\% lo hace con una frecuencia tal, que se considera bullying. Teniendo en cuenta los 3.373 estudiantes que participaron en la investigación, el porcentaje de bullying en los agresores y las agresoras es del 28,11\% (tabla 1). Es de anotar que en el análisis de las variables grado y género no se hallaron correlaciones significativas. De igual manera, se encontró que a medida que aumenta el grado de escolaridad disminuye el índice de bullying.

Tabla 1

Indice de bullying - agresor

\begin{tabular}{lcc}
\hline $\begin{array}{c}\text { Frecuencia } \\
\text { de la agresión }\end{array}$ & $\begin{array}{c}\text { Número de } \\
\text { estudiantes } \\
\text { que agreden }\end{array}$ & $\begin{array}{c}\text { Porcentaje } \\
(\%)\end{array}$ \\
\hline Ocasionalmente & 1.020 & 30,24 \\
Frecuentemente (bullying) & 948 & 28,11 \\
Nunca & 1.405 & 41,65 \\
Total & 3.373 & 100 \\
\hline
\end{tabular}

Nota. Elaboración propia.

\section{Aspectos de las víctimas}

A continuación, se describirán las respuestas del cuestionario que tienen en cuenta la población que expresa haber recibido algún tipo de agresión por parte de un compañero o compañera de clase. En total, se encontró que el 63,30\% de los y las participantes expresaron haber sido víctimas de agresión. Entre las formas más frecuentes de agresión están las ridiculizaciones, con un $33,82 \%$ (tabla 2), principalmente por medio de apodos, burlas e insultos. Además de ello, el 48,01\% ha sido agredido en presencia de otras personas y un $47,03 \%$ ha sido agredido por varias personas a la vez.

En cuanto a los lugares donde ocurre la agresión, el lugar con mayor índice de conductas agresivas es el salón de clase con un 41,31\% (tabla 3). Lo que hace la víctima luego de haber recibido la agresión es, en primera instancia, no contarlo $(17,38 \%)$, decirle a un profesor $(15,50 \%)$, decirle a un amigo $(11,01 \%)$ o decirle a un familiar (10,54\%).
Tabla 2

Formas frecuentes de ser agredido

\begin{tabular}{lcc}
\hline $\begin{array}{c}\text { Formas de } \\
\text { agresión }\end{array}$ & $\begin{array}{c}\text { Número de estudiantes } \\
\text { víctimas }\end{array}$ & $\begin{array}{c}\text { Porcentaje } \\
(\%)\end{array}$ \\
\hline Golpizas & 267 & 12,51 \\
Amenazas & 285 & 13,35 \\
Exclusiones & 110 & 5,15 \\
Ridiculizaciones & 722 & 33,82 \\
Dos o más & 528 & 24,73 \\
No ha sido & 223 & 10,44 \\
agredido & & \\
Total & 2.135 & 100 \\
\hline
\end{tabular}

Nota. Elaboración propia.

Tabla 3

Lugar donde ocurre la agresión

\begin{tabular}{lcc}
\hline \multicolumn{1}{c}{ Lugar } & $\begin{array}{c}\text { Número de estudiantes } \\
\text { agredidos }\end{array}$ & $\begin{array}{c}\text { Porcentaje } \\
(\mathbf{\%})\end{array}$ \\
\hline Baño del colegio & 41 & 1,92 \\
Entrada del & 86 & 4,03 \\
colegio & 221 & \\
Otro & 110 & 10,35 \\
Pasillo del colegio & 158 & 5,15 \\
Patio del colegio & 882 & 7,40 \\
Salón de clase & 61 & 41,31 \\
Zona de deporte & 576 & 2,86 \\
No responde & 2.135 & 26,98 \\
Total & & 100,00 \\
\hline
\end{tabular}

Nota. Elaboración propia.

Del total de estudiantes que ha sido víctima de bullying, el 56,53\% ha sido agredido por lo menos una vez al mes, es decir, frecuentemente. De los 3.373 estudiantes que componen el total de la muestra, 1.207 estudiantes, es decir, el 35,78\% dan cuenta del índice de bullying en la población caracterizada como víctima (tabla 4).

Tabla 4

Indice de bullying - víctimas

\begin{tabular}{lcc}
\hline Frecuencia de la agresión & $\begin{array}{c}\text { Número de } \\
\text { estudiantes agredidos }\end{array}$ & $\begin{array}{c}\text { Porcentaje } \\
(\%)\end{array}$ \\
\hline Ocasionalmente & 928 & 27,51 \\
Frecuentemente (bullying) & 1.207 & 35,78 \\
Nunca & 1.161 & 34,42 \\
No responden & 77 & 2,29 \\
Total & 3.373 & 100,00 \\
\hline
\end{tabular}

Nota. Elaboración propia. 
En el análisis de las variables edad y género, sólo se hallaron diferencias en el grupo de edad de 9 a 11 años, presentándose con mayor frecuencia en las niñas que en los niños. Del total de estudiantes que alguna vez ha agredido a un compañero o compañera de clase, el $84,5 \%$ también ha sido víctima de agresión por otro compañero. De los estudiantes que agreden con frecuencia, el $46,3 \%$ también son víctimas con frecuencia.

\section{Aspectos de los testigos}

Finalmente, como valor agregado en la investigación, se hizo una adaptación al test inicial que sólo tenía en cuenta la posición de los agresores y las víctimas, y se contempló la posición de quienes han sido testigos de la agresión. Así, se logra resaltar la representación de quienes han sido testigos al momento de analizar o pensar este fenómeno. Entre los y las participantes, se encontró que un $71,81 \%$ ha sido testigo de conductas violentas en el ámbito escolar. Las formas más frecuentes de agresión que los testigos han presenciado hacen referencia no a una sola forma de agresión, sino a varias a la vez $(50,83 \%)$, especialmente golpes, burlas, insultos, apodos y empujones (tabla 5). Los y las testigos observan que un $57,23 \%$ de las agresiones son realizadas por varias personas a la vez contra uno o varios de sus compañeros y compañeras. Además de esto, el lugar donde ocurre la mayoría de las agresiones es el salón de clase, con el 44,59\%. Las acciones que realizan los y las testigos después de la agresión observada son: no decirle a nadie $(28,9 \%)$, decirle a un profesor $(18,54 \%)$, decirle a un amigo (13,83\%), decirle a un familiar $(7,93 \%)$.

Tabla 5

Formas frecuentes de agresión

\begin{tabular}{lll}
\hline \multicolumn{1}{c}{ Tipo de agresión } & Frecuencia & Porcentaje (\%) \\
\hline Golpes & 246 & 10,16 \\
Empujones & 106 & 4,38 \\
Insultos & 147 & 6,07 \\
Apodos & 132 & 5,45 \\
Amenaza con armas & 17 & 0,70 \\
Amenaza con golpes & 37 & 1,53 \\
Amenaza de otro tipo & 12 & 0,50 \\
Exclusión & 27 & 1,11 \\
Burlas & 162 & 6,69 \\
Otro & 30 & 1,23 \\
No responde & 275 & 11,35 \\
Dos o más de las anteriores & 1.231 & 50,83 \\
Total & 2.422 & 100,00 \\
\hline
\end{tabular}

Nota. Elaboración propia.
Del total de estudiantes que han sido testigos de bullying, lo ha visto con frecuencia un 63,96\%, y de los 3.373 estudiantes que componen la muestra completa, el 45,92\% dan cuenta del índice de bullying observado por los testigos (tabla 6).

Tabla 6

Indice de bullying - testigo

\begin{tabular}{lcc}
\hline Frecuencia de la agresión & $\begin{array}{c}\text { Número de } \\
\text { testigos }\end{array}$ & $\begin{array}{c}\text { Porcentaje } \\
(\%)\end{array}$ \\
\hline Ocasionalmente & 873 & 25,88 \\
Frecuentemente & 1.549 & 45,92 \\
Nunca & 716 & 21,23 \\
No responden & 235 & 6,97 \\
Total & 3.373 & 100,00 \\
\hline
\end{tabular}

Nota. Elaboración propia.

\section{Discusión y conclusiones}

La agresión y la violencia entre pares que están en su proceso de formación educativa es un fenómeno social que preocupa a docentes, directivos y padres de familia que buscan constantemente que los centros educativos sean espacios de formación integral. La preocupación aumenta cuando la agresión se hace constante y toma diversas formas de manifestación.

Los resultados de este estudio evidencian lo encontrado en otras investigaciones en cuanto a la dificultad del idioma español para denominar el fenómeno del bullying. Sobre este tema, la investigación de Ortega, del Rey y Mora-Merchán (2001) sugiere que quienes se han visto implicados de alguna manera en actos de violencia escolar la nombran de acuerdo con el contexto y la forma de la agresión. Como se expresó en los resultados, muchas de las maneras de nombrar a quienes ejercen bullying denotan y dan poder desde el lenguaje a quienes cometen abuso escolar y se normaliza la violencia como una forma válida de interacción y de dominación, hecho que ha sido documentado en diferentes estudios de las ciencias sociales argumentado desde la sociología del conocimiento, la cognición social, el interaccionismo simbólico, el construccionismo social y el aprendizaje social de Bandura, citado por Fernández (2009). Asimismo, como esta violencia que se vive en las aulas ha pasado a formar parte del trato común entre los alumnos, ha dejado de ser motivo de asombro y en cierta medida ha comenzado a aceptarse como algo "normal", lo que facilita que la violencia escolar se perpetúe (Gómez, 2005). No obstante, la mayoría de los estudiantes rechaza estas 
conductas y reconoce su valencia negativa; así, desde el lenguaje, denotan que quienes agreden se comportan de un modo que afecta la convivencia y las relaciones interpersonales. De esta manera, aunque no fue posible encontrar una palabra que permitiera denominar el bullying entre los hablantes del español, sí se puede observar desde el lenguaje mismo el grado de afinidad o no, el grado de aceptación o no, e incluso un intento de explicación de por qué los estudiantes se comportan así. Como sugerencia, debería ampliarse en futuras investigaciones para aportar a la comprensión social del fenómeno.

Además, esta investigación permite centrar la atención en tres poblaciones: agresores, víctimas y testigos. De la primera, es importante resaltar que casi el $60 \%$ de los estudiantes reconoce haber agredido alguna vez a un par en el ámbito escolar, y aunque la mayoría de los estudiantes reconoció sentirse culpable o preocupado tras la agresión, es significativo que muchos de ellos manifestaron sentirse satisfechos, lo que da cuenta de que, aunque es inadecuado agredir, da satisfacción descargar las emociones y sentirse superior a los demás. Esto, de una u otra manera, normaliza entre estos estudiantes la agresión. Asimismo, se reconfiguran las representaciones sociales creadas en los centros educativos develando discursos que objetivan la intimidación, el maltrato y el acoso; además de ello, la forma de intervención ante las situaciones de agresión, la falta de pertinencia en la norma y la forma como muchas personas reaccionan ante la agresión inciden en la creación de una sociedad que se acostumbra a soportar estas formas de relación dentro de la cotidianidad (Guiso y Ospina, 2010).

En el presente estudio, el porcentaje de estudiantes que reconocieron ser víctimas de algún tipo de agresión en el ámbito escolar de manera frecuente fue de más de la tercera parte, un dato más alto que en otras investigaciones similares (Paredes et al., 2008); y aunque esta investigación no se centró en las consecuencias del bullying, sí sorprende el alto porcentaje de víctimas, ya que varias investigaciones concuerdan en que estas terminan exhibiendo un profundo malestar psicológico y emocional del cual la ideación suicida, el bajo rendimiento académico y la deserción escolar son más frecuentes que en quienes no han sido víctimas de bullying (Rigby, 2003). Finalmente, los testigos, aunque no son actores ni víctimas, influyen desde su actividad o pasividad a que la agresividad aumente o disminuya. Por ello, se añadió al cuestionario inicial un apartado que hablara de la percepción de los testigos y aunque tuvo como limitante que no se realizó una prueba piloto de las preguntas, sí se dio continuidad al formato adoptado en el cuestionario realizado para víctimas y agresores; de esta manera, se encontró que más del $70 \%$ de los estudiantes que respondieron la encuesta han sido testigos de agresión en sus centros educativos. Es de reconocer que aunque no tomen la iniciativa en las agresiones sí participan de ellas, y son a quienes Olweus (1998) denominó como agresores pasivos.

En las formas más frecuentes de agresión, se conserva la tendencia percibida en los estudios de otros países, en los que se encontró que la manera más común es de naturaleza verbal, específicamente en la forma de ridiculización o apodos (Paredes et al., 2008). Este hecho influye en que por ello se presente fácilmente en el aula de clase. El salón de clases es el lugar donde sucede la mayoría de las conductas violentas, hecho que se ha observado en otras investigaciones como en la de Hoyos, Aparicio y Córdoba (2005) y en la de Uribe, Orcasita y Aguillón (2012). Por tal, son los lugares que requieren mayor atención y vigilancia, hecho que fue señalado en la investigacion realizada en el área metropolitana de Medellín, como la falta de pertinencia en las normas y en los modos de comportamiento del adulto educador, la cual, a su vez, es leída por niños, niñas y jóvenes como falta de interés y débil control de los responsables frente a la intimidación, la amenaza o el acoso en la escuela (Guiso y Ospina, 2010).

Ante esto, las víctimas y los testigos deciden, como primera opción, no contarle a nadie, lo cual evidencia dificultades en la comunicación, la manifestación de emociones y hace posible que la víctima desarrolle una mayor percepción de impotencia, y en quienes agreden una mayor sensación de satisfacción. Los resultados dan cuenta de la importancia de que los diferentes miembros de la comunidad educativa ayuden a contrarrestar el problema. Ya sea porque víctimas y testigos no cuenten o porque los adultos no intervengan, hay un alto porcentaje de estudiantes que no reciben consecuencias tras agredir a un compañero o compañera, $y$ sólo alrededor de la tercera parte de quienes han agredido han recibido una consecuencia de carácter institucional (llamado de atención, ser llevado a las directivas, anotación en la hoja de vida, citación del acudiente, entre otras). Este es un factor que hace que las conductas de bullying vuelvan a ocurrir y además genera en víctimas y testigos un sentimiento de impunidad.

Otro aspecto para resaltar es que más de la mitad de las agresiones suceden en presencia de otras personas y, en muchos de los casos, son más de dos personas los que agreden. Esto muestra que el bullying es un 
fenómeno social, lo cual concuerda con otros estudios donde se habla de que en los lugares donde hay menos público, no es tan frecuente que se dé el acoso escolar (Rodríguez, 2011). Este factor hace que si los testigos apoyan o guardan silencio ante el maltrato entre pares, entonces, este se mantiene.

Aunque este estudio no evaluó las causas del bullying, sí nos permite reflexionar sobre algunos factores que pueden favorecer la violencia escolar: la regulación de emociones, la vigilancia en el aula de clase, el hecho de que víctimas y testigos no cuenten cuando hay agresión y el hecho de no siempre presentarse consecuencias tras la agresión. Ante esto, es de resaltar que la prevención del bullying es un asunto de corresponsabilidad, en el que escuela, familia y Estado deben aunar esfuerzos para disminuir la presencia de este fenómeno. Además, es importante incrementar investigaciones que traten el tema, no sólo desde su diagnóstico e incidencia, sino también de estrategias eficaces de intervención, siendo este trabajo un indicio para futuras investigaciones, ya que estos resultados crean la necesidad de seguir inquiriendo y profundizando sobre todo aquello que afecte a la población escolarizada, por medio de la aplicación de los resultados de este estudio. Entre ellos, es importante continuar realizando investigaciones sobre la forma que la población hispanoparlante denomina el bullying; la relación entre los estudiantes que son tanto víctimas como agresores y la incidencia de los testigos.

Si se orienta sobre el bullying, sus consecuencias y cómo contrarrestarlo, toda la comunidad educativa trabajará en pos de denunciarlo, repudiarlo y, por ende, disminuirlo. Es importante fomentar en los educandos el reconocimiento de su responsabilidad en el inicio, mantenimiento y promoción del bullying, así como en su intervención y promoción. Porque la transformación de la educación y la cultura propician la convivencia, tal como se encontró en esta investigación cuando los resultados mostraron que al aumentar el grado de escolaridad disminuyen los índices de agresión (Hoyos, Aparicio y Córdoba, 2005).

\section{Referencias}

Ávila, J. H., Osorio, L., Cuello, K., Cogollo, N. y Causado, K. (2010). Conducta bullying y su relación con la edad, género y nivel de formación en adolescentes. Revista Psicogente, 13(23), 13-26.

Avilés, J. M. (2003a). Bullying: intimidación y maltrato entre el alumnado. Bilbao: Stee-Eilas.
Avilés, J. M. (2003b). El maltrato entre escolares en el contexto de las conductas de acoso. Bullying en la escuela: modelos de intervención. En J. M. Avilés (Dir.). Riesgos psicosociales en la enseñanza (p. 7). Valladolid: Confederación de sTEs-intersindical.

Carrera, M. V., De Palma, R. y Lameiras, M. (2011). Toward a More Comprehensive Understanding of Bullying in School Settings. Educational Pychology Review, 23(4), 479-499.

Camargo, M. (2012, 15 de marzo). Crece el acoso vía internet entre escolares en Colombia. Revista Semana. Recuperado de: http://www.semana.com/nacion/crece-acosovia-internet-entre-escolares-colombia/173838-3.aspx

Chaux, E. (2011). Múltiples perspectivas sobre un problema complejo: comentarios sobre cinco investigaciones en violencia escolar. Psykhe, 20(2), 79-86.

Ministerio de Educación Nacional. (2013, 11 de septiembre). Decreto 1965. Bogotá, Colombia: MEN.

Fernández, I. (2009). Justificación y legitimación de la violencia en la infancia. Un estudio sobre la legitimación social de las agresiones en los conflictos cotidianos entre menores (Disertación doctoral no publicada). Universidad Complutense de Madrid, España.

Gómez, N. (2005). Violencia e institución educativa. Revista Mexicana de Investigación Educativa, 10(26), 671-693.

Guiso, A. y Ospina, V. (2010). Naturalización de la intimidación entre escolares: un modo de construir lo social. Revista Latinoamericana de Ciencias Sociales, Niñez y Juventud, 8(9), 535-556.

Hoyos, O., Aparicio, J. y Córdoba, P. (2005). Caracterización del maltrato entre iguales en una muestra de colegios de Barranquilla (Colombia). Psicología desde el Caribe, $16,1-28$.

Congreso de Colombia. (2013, 15 de marzo). Ley 1620. Bogotá, Colombia: Congreso de la República.

Olweus, D. (1998). Conducta de acoso y amenaza entre escolares ( $4^{\mathrm{a}}$ ed.). Madrid: Morata, S. L.

Olweus, D. (2001). Peer Harassment. A Critical analysis and some Important Issues. En J. Juvonen y S. Graham (Eds.), Peer Harassement in school (pp. 3-20). New York: Guilford Press.

Ortega, R., del Rey, R. y Mora-Merchan, J. (2001). Violencia entre escolares: conceptos y etiquetas verbales que definen el fenómeno del maltrato entre iguales. Revista Interuniversitaria de Formación del Profesorado, 41, 95-113.

Osorio, C. (2009). Relación entre el tiempo de exposición a la televisión y la presentación de conductas agresivas entre pares (bullying) de un grupo de estudiantes en un contexto escolar de la ciudad de Cali (Trabajo de grado para optar al título de psicóloga). Universidad Antonio Nariño, Colombia. 
Paredes, M., Álvarez, M., Lega, L. y Vernon, A. (2008). Estudio exploratorio sobre el fenómeno del "Bullying" en la ciudad de Cali, Colombia. Revista Latinoamericana de Ciencias Sociales, Niñez y Juventud, 6(1), 295-317.

Paredes, M. T., Lega, L., Cabezas, H., Ortega, M., Medina, Y. y Vega, C. (2011). Diferencias transculturales en la manifestación del bullying en estudiantes de escuela secundaria. Revista Latinoamericana de Ciencias Sociales, Niñez y Juventud, 9(2), 761-768.

Rigby, K. (2003, octubre). Consequences of Bullying in schools. The Canadian Journal of Psychiatry, 48(9), 583-590.
Rodríguez, M. G. (2011). Bullying (Acoso escolar). Instituto Mexico Primaria. Recuperado de: http://www.maristas.org.mx/portal/sites/default/files/Material_de_Bullying_psic_ma_guadalupe.pdf

Rodríguez, A. y Mejía, Y. (2012). Bullying: un fenómeno por transformar. Duazary, 9(1), 98-104.

Uribe, A. F., Orcasita, L. T. y Aguillón, E. (2012). Bullying: redes de apoyo social y funcionamiento familiar en adolescentes de una institución educativa de Santander. Psychologia. Avances de la disciplina, 6(2), 83-99. 